Editorial

\title{
Discriminators for optic neuropathy and maculopathy
}

\section{Editorial}

Optic neuropathies and maculopathies are more common causes of visual loss. However, as optic neuropathies and maculopathies can share some common characteristics, the overlapping of the features of these pathologies occur sometimes and this can cause the difficulty in diagnosis and even the important challenges in the management of the underlying exact pathology. The early diagnosis of some neuroophthalmological emergencies such as arteritic anterior ischemic optic neuropathy (AION) and optic neuritis is critical. There are several discriminator aspects providing to distinguish between optic neuropathies and maculopathies. ${ }^{1-9}$ The comparison of all aspects and discriminators of optic neuropathy and maculopathy was given in Table 1.To apply the highlights and discriminators given in table will facilitate to distinguish the optic nerve disease from macular diseases.
Volume 7 Issue 7 - 2017

Burak Turgut,' Fatoș Altun Turgut ${ }^{2}$

'Department of Ophthalmology, Yuksek Ihtisas University, Turkey ${ }^{2}$ Elazig Training and Research Hospital, Turkey

Correspondence: Burak Turgut, Professor of Ophthalmology, Yukseklhtisas University, Faculty of Medicine, Department of Ophthalmology, 06520, Ankara, Turkey, Tel +90 312 280360I, Emailburakturgut@yiu.edu.tr

Received: December 08, 2017 | Published: December II, 2017

Table I The comparison of all aspects of optic neuropathy and maculopathy ${ }^{1-9}$

\begin{tabular}{|c|c|c|}
\hline Feature & Optic nerve disease & Macular disease \\
\hline Onset & Variable (often acute, subacute) & Variable \\
\hline Course & Variable (Progressive, transient, or stable) & Mostly slower progression \\
\hline Visual experience & Shading, clouding, graying, darkening & Central blurring, glaring, photophobia, metamorphopsia \\
\hline Ocular pain & Maybe occur in eye movements & No usual \\
\hline Refractive change & No usual & Maybe develop hyperopic shift \\
\hline VA loss & Variable reduction, even to NLP level & Significantly reduction but not to NLP \\
\hline Pupils & RAPD in case of unilateral or asymmetrical ON disease & No RAPD, if severe unilateral retinal involvement is absent \\
\hline CS and brightness & Significantly reduced & Mildly reduced \\
\hline Color vision & Significantly reduced & Mildly reduced \\
\hline VF defect & Variable (Central, centrocecal, altitudinal, blind spot enlargement) & Central scotoma \\
\hline Amsler Grid & Variable scotoma & Central scotoma, metamorphopsia \\
\hline Pulfrich phenomena & Novaluable & Central macular disease (hole, cyst) \\
\hline PSR time & Normal (under 30 sn) & Prolonged recovery time (over $90 \mathrm{sn}$ ) \\
\hline VER & Abnormal (Large delayed latency and decreased amplitude) & Normal or mildly abnormal (small latency delay) \\
\hline ERG & Normal & $\begin{array}{l}\text { Full field ERG is often normal while multifocal ERG is } \\
\text { usually abnormal }\end{array}$ \\
\hline FFA & $\begin{array}{l}\text { Late phase disc leakage in optic disc edema, peripapillary filling } \\
\text { delay or ischemia in AION }\end{array}$ & Dependent on cause of maculopathy \\
\hline FAF & Hyperautoflorescence (ON drusen, astrocytic hamartoma) & Variable depend on macular disease \\
\hline \multirow[b]{2}{*}{ SD-OCT } & $\begin{array}{l}\text { Increasing or decreasing in RNFL thickness (edema or atrophy) } \\
\text { Increasing in peripapillary RNFL thickness (myelinated nerve fiber }\end{array}$ & $\begin{array}{l}\text { Vitreo-retinal interface abnormalities } \\
\text { Various intra- or sub-retinal/sub-RPE pathologies in macular } \\
\text { region }\end{array}$ \\
\hline & $\begin{array}{l}\text { or cotton wool spot) } \\
\text { Decreasing macular GCC (optic neuritis) }\end{array}$ & $\begin{array}{l}\text { Altering macular retina thickness } \\
\text { Altering macular choroid thickness } \\
\text { Decreasing macular GCC (antimalarial maculopathy) }\end{array}$ \\
\hline
\end{tabular}

VA, visual acuity; NLP, no light perception; RAPD, relative afferent pupillary defect; CS, contrast sensitivity; VF, visual field; PSR, photo-stress recovery; ON, optic nerve; ERG, electroretinogram; FFA, fundus fluorescein angiography; FAF, fundus auto-fluorescence;AION, anterior ischemic optic neuropathy; SD-OCT, spectral domain-optical coherence tomography; RNFL, retinal nerve fiber layer; GCC, ganglion cell complex;VER, visual evoked response; RPE, retina pigment epithelium 


\section{Acknowledgments}

None.

\section{Conflicts of interest}

The author declares that there is no conflict of interest regarding the publication of this paper.

\section{Funding}

The author received no financial support for the research, authorship, and/or publication of this article.

\section{References}

1. Nagia L, Eggenberger E. Differentiating retinal from optic nerve syndromes. Curr Opin Ophthalmol. 2013;24(6):528-533.

2. Sadun AA. Distinguishing between clinical impairments due to optic nerve or macular disease. Metab Pediatr Syst Ophthalmol. 1985;13(24):79-84.
3. Holder GE. Electrophysiological assessment of optic nerve disease. Eye (Lond). 2004;18(11):1133-1143.

4. Janáky M. The Roles of Electroretinography (ERG) and Visual Evoked Potential (VEP) Examinations in the Diseases of the Retina and/or the Optic Nerve. In: Somlai J, Kovács T, editors, Neuro-Ophthalmology. Springer. 2016;325-331.

5. Rebolleda G, Diez-Alvarez L, Casado A, et al. OCT: New perspectives in neuro-ophthalmology. Saudi J Ophthalmol. 2015;29(1):9-25.

6. Hasrod N, Rubin A. Defects of colour vision: A review of congenital and acquired colour vision deficiencies. Afr Vision Eye Health. 2016;75:a365.

7. Simunovic MP. Acquired color vision deficiency. Surv Ophthalmol. 2016;61(2):132-155

8. Shin SY, Kim DS, Ko MK. Fluorescein angiographic features of choroidal insufficiency in anterior ischemic optic neuropathy. Korean $J$ Ophthalmol. 1999;13:100-104.

9. Yung M, Klufas MA, Sarraf D. Clinical applications of fundus autofluorescence in retinal disease. Int J Retin Vitr. 2016;2:12. 